\title{
Primary endobronchial plasmacytoma involving local lymph nodes and presenting with rare immunoglobulin G lambda monoclonal gammopathy
}

\author{
Sen Wei MD, Xin Li MD, Zuoqing Song MD, Honglin Zhao MD, \\ Xiaomin Qiu MD, Lei Gong MD, Jun Chen MD PhD, Qinghua Zhou MD
}

\begin{abstract}
S Wei, X Li, Z Song, et al. Primary endobronchial plasmacytoma involving local lymph nodes and presenting with rare immunoglobulin G lambda monoclonal gammopathy. Can Respir J 2012;19(3):e28e30.

Extramedullary plasmacytoma occurring as a primary pulmonary lesion is rare. The present report describes a 42-year-old Chinese man diagnosed with primary pulmonary plasmacytoma following left lower lobectomy. Of note, an extremely rare immunoglobulin G lambda paraprotein was documented in the patient's serum by immunofixation electrophoresis. The patient has been well, showing no local recurrence or multifocal disease during a 15-month follow-up.
\end{abstract}

Kew Words: Extramedullary plasmacytoma; Lobectomy; Primary pulmonary plasmacytoma

\begin{abstract}
Cxtramedullary plasmacytoma (EMP) is an uncommon and typically $\mathrm{E}_{\text {progressive tumour capable of originating in any body part outside }}$ the bone marrow. Collectively, such growths account for $<5 \%$ of all plasma cell neoplasms (1). While more than $80 \%$ of EMPs arise in the upper respiratory or digestive tract, primary pulmonary plasmacytoma (PPP) is actually quite rare. To our knowledge, at most, 30 cases have been documented to date in the English literature (2). These tumours seldom produce paraprotein, but those that do are generally associated with immunoglobulin (Ig) G kappa monoclonal gammopathy. In the present article, we describe a primary endobronchial plasmacytoma (PEB) of the left lower lobe with local lymph node involvement, treated by lobectomy and systematic mediastinal lymphadenectomy. The histological impression was confirmed by immunohistochemistry, but there was clinical evidence of multiple myeloma (MM). However, an extremely rare IgG lambda paraprotein was documented in the patient's serum by immunofixation electrophoresis.
\end{abstract}

\section{CASE PRESENTATION}

A 42-year-old Chinese man was admitted with radiographic evidence of a peripheral left lung lesion, suspicious of primary lung cancer. The mass, discovered on routine examination, was asymptomatic by account. The patient denied fever, cough, wheezing, hemoptysis, or other systemic or neurological symptoms, although he did claim a 20 -year smoking history of approximately 20 to 25 cigarettes/day. There was no family history of lung cancer and a review of systems was noncontributory. On admission, peripheral blood count, serum chemistry and urinalysis were normal.

\section{Radiological findings}

An enhanced chest computed tomography (CT) scan revealed a $2.1 \mathrm{~cm}$ $\times 2.3 \mathrm{~cm} \times 3.5 \mathrm{~cm}$ mass in the left lower lobe with mediastinal and hilar lymphadenopathy. The lesion was oval, fairly circumscribed and uniform in appearance (Figure 1A). At the same time, an incidental

\author{
Un plasmacytome endobronchique primitif touchant \\ les ganglions lymphatiques et s'accompagnant d'une \\ rare gammopathie monoclonale lambda de \\ l'immunoglobuline $\mathrm{G}$
}

Il est rare qu'un plastocytome extramédullaire se présente sous forme de lésion pulmonaire primitive. Le présent rapport décrit le cas d'un Chinois de 42 ans recevant un diagnostic de plasmacytome pulmonaire primitif après une lobectomie inférieure gauche. Il est à souligner qu'on a constaté une paraprotéine lambda de l'immunoglobuline G d'une extrême rareté dans le sérum du patient par électrophorèse d'immunofixation. Le patient est bien et n'a pas présenté de récurrence locale ou de maladie multifocale dans le cadre d'un suivi de 15 mois.

endobronchial nodule was found in the posterior basal segmental bronchus of the left lower lobe, obstructing the distal bronchus (Figure 1B). On bronchoscopy, this was a solid neoplasm, tan-white in colour and with a smooth surface (Figure 1C); however, bronchoscopic biopsy failed to show malignancy or provide a diagnosis.

To exclude malignancy, abdominal CT, magnetic resonance imaging of the brain and skeletal CT were performed, with negative results (the facility did not routinely perform whole-body positron emission tomography-CT scans). Radical left lower lobectomy and systematic mediastinal lymphadenectomy were then performed (March 4,2010 ) through standard posterolateral thoracotomy. Intraoperative frozen sectioning was not performed.

\section{Pathological findings}

Routine sections of the gray-white endobronchial lesion $(0.5 \mathrm{~cm} \times 1.0 \mathrm{~cm}$ $\times 1.2 \mathrm{~cm}$ ) showed moderate size, ovoid plasma cells arranged in sheets, beneath intact respiratory mucosa (Figure 2A). Individually, the cells were well-differentiated, marked by minor nuclear and cytoplasmic variability and few (if any) mitotic figures. This particular growth displayed strong CD138 and CD79a immunostaining (Figures 2B and 2C), but was negative for CD20. Light-chain restricted staining (lambda only) was also demonstrated. Five lymph nodes (two peribronchial [\#11], one subcarinal [\#7], and two at the pulmonary ligament $[\# 9]$ ) contained similar plasmacytic infiltrates. The initially detected parenchymal mass of the left lower lobe was inflammatory in nature and likely resulted from airway obstruction.

There was no evidence of $\mathrm{MM}$ in a subsequent bone marrow biopsy. In fact, the marrow was hypocellular, with only $1.5 \%$ plasma cells. Serum electrophoresis, on the other hand, was compatible with a low monoclonal spike, and a faint restricted band of $\operatorname{IgG}$ lambda appeared against a polyclonal background on immunofixation electrophoresis, but urinary Bence-Jones protein was negative. A final diagnosis of PEB was made.

Department of Lung Cancer Surgery, Tianjin Key Laboratory of Lung Cancer Metastasis and Tumor Microenvironment, Tianjin Medical

University General Hospital, Tianjin China

Correspondence: Dr Jun Chen, Department of Lung Cancer Surgery, Tianjin Key Laboratory of Lung Cancer Metastasis and Tumor

Microenvironment, Tianjin Medical University General Hospital, Tianjin 300052, China. Fax 86-22-60363013,

e-mail huntercj2004@yahoo.com; zhough1016@yahoo.com.cn. 

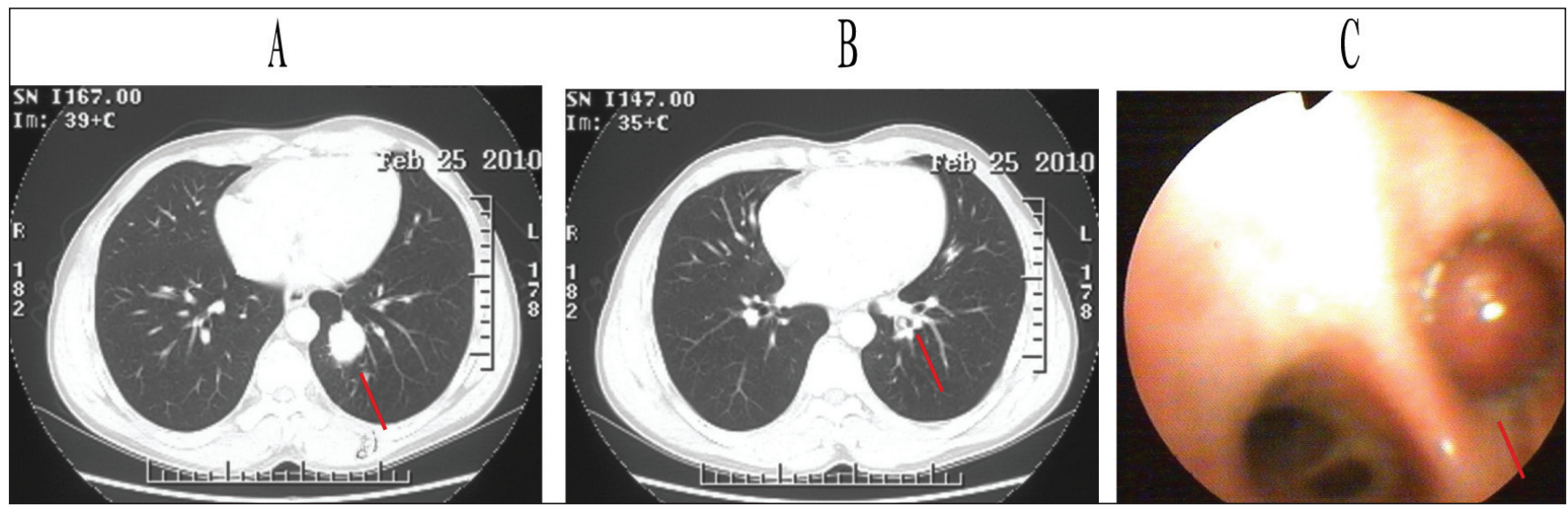

Figure 1) Enhanced chest computed tomography scan (A and B) and bronchoscopic detection (C). Panel A Left lower lobe mass (red line). Panel B Small nodule in basal segment bronchus of left lower lobe (red line). Panel C Smooth, solid nodule in posterior basal segment bronchus of left lower lobe (red line)

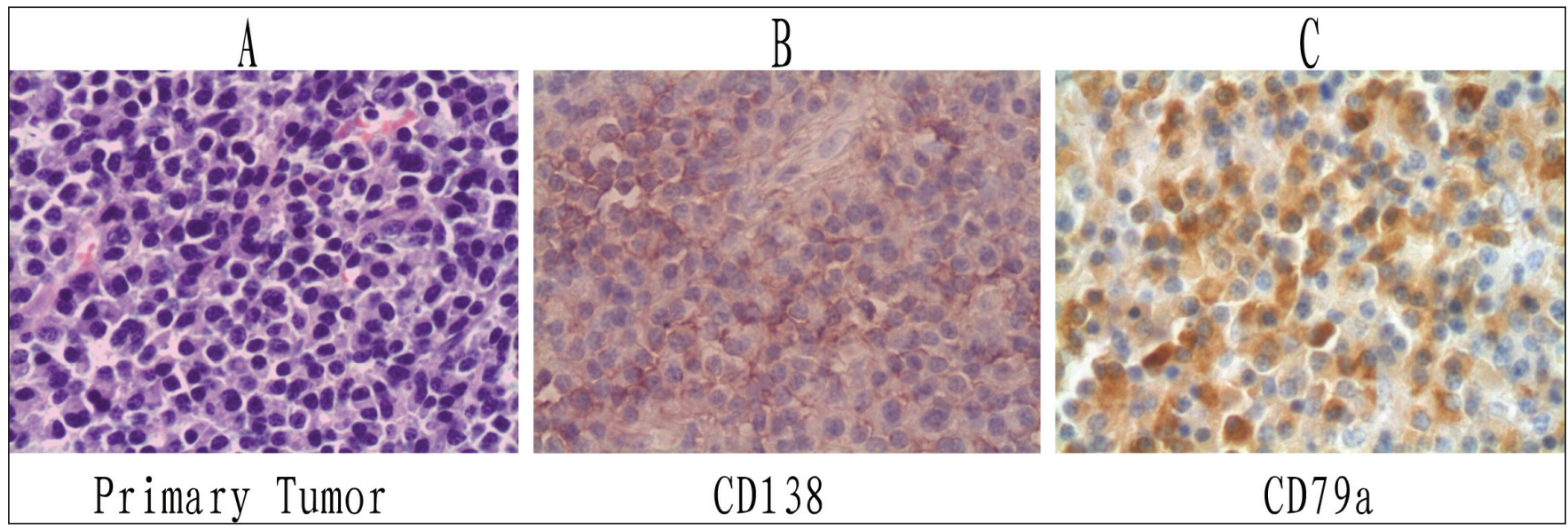

Figure 2) Pathological images (original maginifcation $\times 400$ ). A Hematoxylin and eosin staining of primary endobronchial plasmacytoma. Immunohistochemical staining of primary tumour with antibodies to CD138 (B) and CD79a (C)

After a 15-month follow-up, the patient was well, without evidence of local recurrence or multifocal disease. A repeat bone marrow biopsy 12 months postoperatively was normal (Figure 3) and, on immunofixation electrophoresis, the patient's paraprotein was not apparent.

\section{DISCUSSION}

PPP is a rare tumour of plasma cell origin and a subset of EMP. Two distinct growth patterns are characteristically observed: nodule/mass or pulmonary infiltrate, with or without local lymph node involvement (3-5). Although both lungs are equally affected, upper lobes are more likely to harbour lesions (3). In their study and review of published pulmonary plasmacytomas (supported by immunohistochemistry), Koss et al (3) noted that most patients were middle-age or older, with mean and median age of 57 years and 55 years, respectively. They disagreed with Joseph et al (6) who found PPP more prevalent in a younger group of patients (median age 43 years), contending that the data may have been skewed by including cases of plasma cell granuloma. Cumulative reports indicate that radiographic findings with PPP tend to be nonspecific. Bronchoscopic biopsies are also usually unsatisfactory due to specimen inadequacy, superimposed inflammation or other limitations (eg, submucosal/peripheral mass). Establishing the diagnosis may, therefore, be relatively difficult (6).

Apart from routine histology, immunohistochemistry is required to confirm PPP. To our knowledge, only four cases of PEBs have been described and confirmed with immunostaining $(2,4,7,8)$, so this is an extremely uncommon event (Table 1). All other published cases either reflected a parenchymal lesion in proximity or lacked immunohistochemical support. In the present case, both hematoxylin and eosin preparations and strongly stained CD138 and CD79a markers confirmed the tumour as PPP.

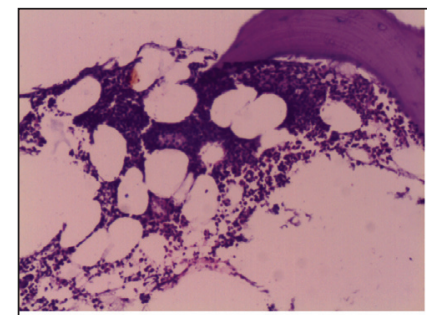

$\mathrm{x} 100$

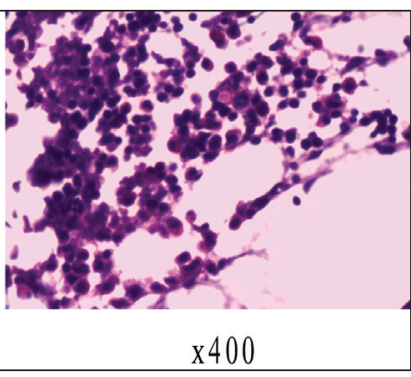

$\mathrm{x} 400$
Figure 3) Bone marrow biopsy (12 months postoperatively)

In $50 \%$ of EMPs, a paraprotein is identified by serum protein electrophoresis, and may also be detected in urine and pleural fluid. The level of paraproteinemia is directly related to tumour burden and clinical course (9). While its disappearance seemingly provides a useful marker of therapeutic success, a persistence or increase after definitive treatment of EMP could suggest systemic disease $(6,10)$. PPP producing IgG lambda $\mathrm{M}$ protein is a rarity. Only three other cases have been described $(6,10,11)$. Our case details the unique association of a primary endobronchial lesion and monoclonal IgG lambda, with only one other account similarly citing a loss of paraprotein after tumour resection. In another case (9), serum and urine immunoelectrophoresis revealed a vague band of monoclonal lambda light chain six months after surgical treatment. Amin also reported one patient who developed an abnormal urinary lambda light chain and serum IgG six months after surgery, when skull lesions appeared. MM later manifested (9). 
TABLE 1

Clinical characteristics in four cases of primary endobronchial plasmacytoma (English publications)

\begin{tabular}{|c|c|c|c|c|c|c|c|c|}
\hline \multirow{2}{*}{$\begin{array}{l}\text { First author } \\
\text { (ref) }\end{array}$} & \multirow{2}{*}{$\begin{array}{l}\text { Age, } \\
\text { years/ } \\
\text { sex }\end{array}$} & \multirow[b]{2}{*}{ Symptoms } & \multirow[b]{2}{*}{ Location } & \multirow[b]{2}{*}{ Treatment } & \multicolumn{4}{|c|}{ Immunohistochemical Lymph node } \\
\hline & & & & & Hematological & findings & status & Follow-up \\
\hline Brackett (7) & 68/male & $\begin{array}{l}\text { Cough, } \\
\text { dyspnea }\end{array}$ & Left mainstem bronchus & Nd-YAG laser & $\begin{array}{c}\text { No M } \\
\text { component }\end{array}$ & Lambda (+) & Normal & Not reported \\
\hline Terzi (8) & 65/male & $\begin{array}{l}\text { Cough, } \\
\text { dyspnea }\end{array}$ & Tracheobronchial carina & $\begin{array}{l}\text { Subtotal resection of the } \\
\text { tracheobronchial carina }\end{array}$ & $\begin{array}{c}\text { No M } \\
\text { component }\end{array}$ & Kappa (+) & Normal & 63 months \\
\hline Piard (4) & 70/male & Dyspnea & $\begin{array}{l}\text { Posterior part of the left } \\
\text { main stem bronchus }\end{array}$ & Left lower lobectomy & $\begin{array}{c}\text { No M } \\
\text { component }\end{array}$ & Kappa (+) & Metastasis & Not reported \\
\hline Edelstein (2) & 47/male & $\begin{array}{l}\text { Cough, } \\
\text { shortness } \\
\text { of breath }\end{array}$ & Left proximal bronchus & $\begin{array}{l}\text { Bronchoscopic debulking } \\
\text { and YAG laser }\end{array}$ & Not reported & Kappa (+) & Not reported & 8 months \\
\hline
\end{tabular}

M Monoclonal protein; Nd-YAG Neodymium-doped yttrium aluminum garnet; + Positive; ref Reference

Although the standard treatment of EMP is radical resection alone or in combination with radiotherapy $(3,4,7)$, optimal treatment of PPP remains controversial due to limited follow-up data on too few patients. For the same reason, the prognosis of PPP remains uncertain; and because the diagnosis is rendered after total resection of the tumour, clinicians are often at a loss with regard to the need for further therapy. Edelstein et al (2) determined that approximately $40 \%$ of reported cases ultimately progress to $\mathrm{MM}$; therefore, close postoperative monitoring is advised, including serial quantitative paraprotein determinations and bone marrow biopsies or whole-body positron emission-CT scans.

Aggressive surgical treatment alone was used for our patient despite the involvement of five local lymph nodes. However, based on a negative bone marrow examination, the hematologist did not recommend chemotherapy. Unlike Edelstein et al (2), we believe that endoscopic resection and laser ablation provide inadequate local control, creating a potential for persistent/recurrent disease and making postoperative radiation mandatory. Furthermore, although radiation is commonly used in primary head and neck plasmacytomas with positive results, the role of radiotherapy for PEB remains uncertain. Our patient did not opt for mediastinal radiotherapy, having undergone local lymph node resection along with tumour excision. He is to be followed with bone marrow biopsy annually to exclude MM.

ACKNOWLEDGEMENTS: This study was partly supported by the grants from Science and Technology Support Key Program of Tianjin (09ZCZDSF04100, 09ZCZDSF04000).

AUTHOR CONTRIBUTIONS: Sen Wei performed the research, analyzed the data and wrote the manuscript; Xin Li, Zuoqing Song, Honglin Zhao, Xiaomin Qiu and Lei Gong collected the data and followed the patient; Jun Chen performed the operation and research, analyzed the data, and wrote the manuscript; Qinghua Zhou performed the operation, and revised the manuscript.

DISCLOSURES: The authors have no financial disclosures or conflicts of interest to declare.

\section{REFERENCES}

1. GalieniP,Cavo M,Pulsoni A, et al. Clinical outcome of extramedullary plasmacytoma. Haematologica 2000;85:47-51.

2. Edelstein E, Gal AA, Mann KP, Miller JI, Mansour KA. Primary solitary endobronchial plasmacytoma.

Ann Thorac Surg 2004;78:1448-9.

3. Koss MK, Hochholzer L, Moran GA, et al. Pulmonary plasmacytomas: A clinicopathologic and immunohistochemical study of five cases. Ann Diagn Pathol 1998;2:1-11.

4. Piard F, Yaziji N, Jarry O, et al. Solitary plasmacytoma of the lung with light chain extracellular deposits: A case report and review of the literature. Histopathology 1998;32:356-61.

5. Wise JN, Schaefer RF, Read RC. Primary pulmonary plasmacytoma: A case report. Chest 2001;120:1405-7.

6. Joseph G, Pandit M, Korfhage L. Primary pulmonary plasmacytoma. Cancer 1993;71:721-4.

7. Brackett LE, Myers JR, Sherman CB. Laser treatment of endobronchial extramedullary plasmacytoma. Chest 1994106:1276-7.

8. TerziA, FurlanG, ZannoniM,et al. Endobronchial extramedullary plasmacytoma. Report of one case. Lung Cancer 1996;16:95-100.

9. Amin R. Extramedullary plasmacytoma of the lung. Cancer 1985;56:152-6.

10. Chang CC,Chang YL, Lee LN, et al. Primary pulmonary plasmacytoma with immunoglobulin G/lambda light chain monoclonal gammopathy. J Thorac Cardiovasc Surg 2006132:984-5.

11. Shaikh G, SehgalR, Mehrishi A, et al. Primary pulmonary plasmacytoma. J Clin Oncol 2008;26:3089-91. 


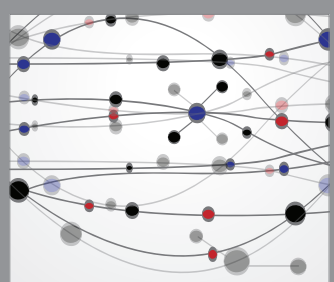

The Scientific World Journal
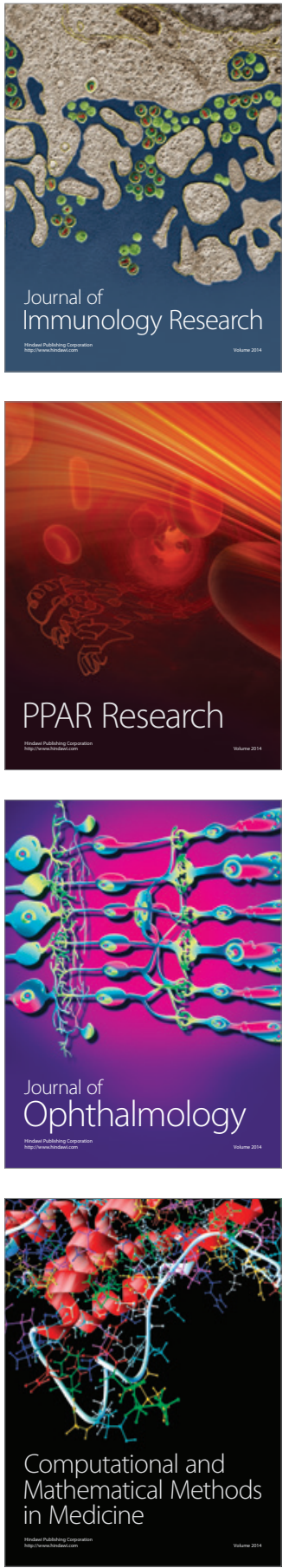

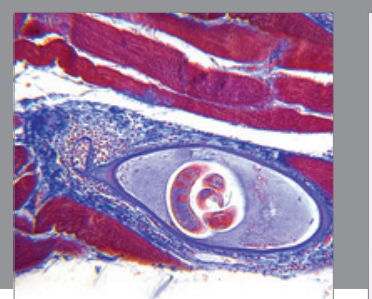

Gastroenterology Research and Practice

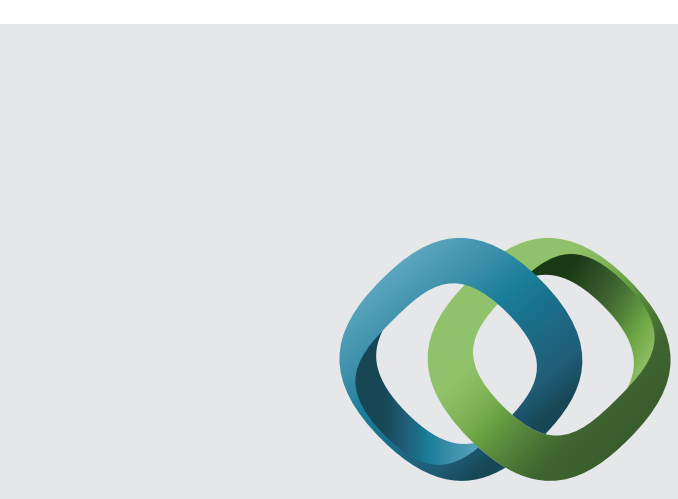

\section{Hindawi}

Submit your manuscripts at

http://www.hindawi.com
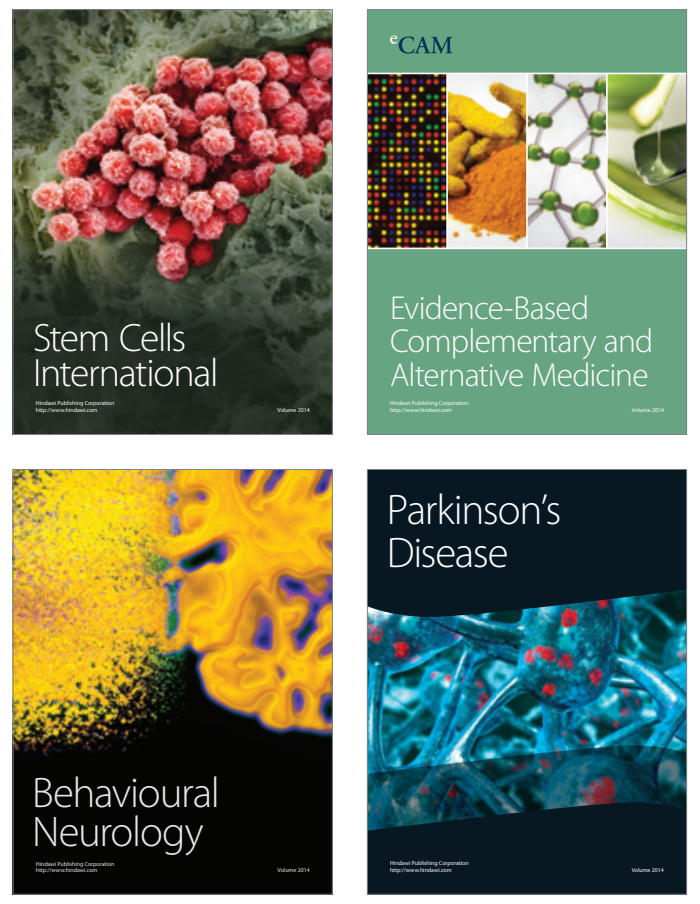
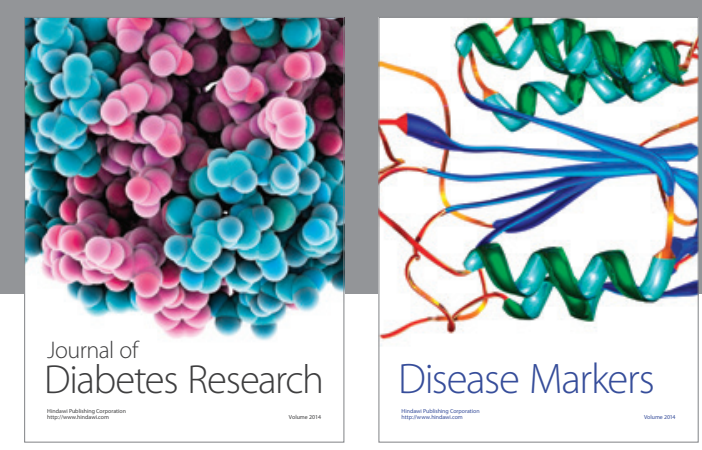

Disease Markers
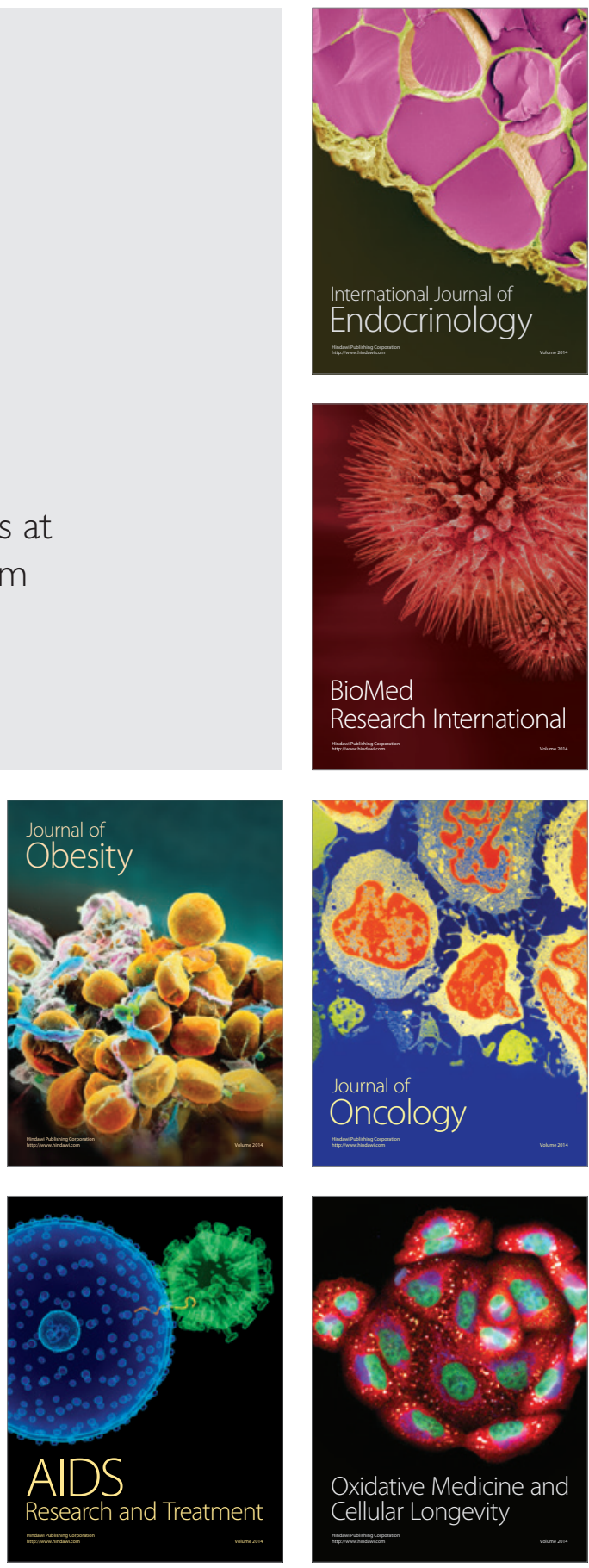